\title{
GWAS between flax accessions and agronomically important phenotypic traits
}

Sokolkova A. ${ }^{1}$, Rozhmina T. ${ }^{2}$, Kanapin A. ${ }^{1}$, Samsonova A. ${ }^{1}$, Surkova S. ${ }^{1}$, Bankin M. ${ }^{1}$, Samsonova M. ${ }^{1}$

${ }^{1}$ Peter the Great St. Petersburg Polytechnic University, St. Petersburg, Russia

${ }^{2}$ Federal Research Centre for Bast Fiber Crops, Torzhok, Russia

*email: alyonasok@yandex.ru

Flax is one of the most important crops, both in Russia and in other countries. In modern conditions, fibre flax is considered as a strategic crop in Russia, since it is able to replace raw cotton, which has moved into the category of imported raw materials. Oil flax is used for the production of seeds, which are characterized by valuable nutritional and therapeutic properties. The flax collection created at the Federal Research Centre for Bast Fiber Crops is one of the largest in the world and covers almost all the genetic diversity of the crop. The collection was screened for resistance to Fusarium wilt and highly resistant samples were identified. Here we present the results of GWAS for 306 accessions from this collection. We performed 8X depth genome sequencing for accessions and called 3,416,829 SNPs. Further SNP filtering was implemented with inclusion criteria: minor allele frequency (MAF) more than 3\%, genotype call-rate more than $90 \% .49,621$ SNPs passed all filters and remained for further analysis. Phenotyping of 306 accessions was performed three times at experimental station in Torzhok, Russia: in 2019 and two times in 2020. The measured traits are plant height, length of technical part of the stem, technical part weight, inflorescence length, number of bolls, seeds per plant, 1000 seed weight, the diameter of the stem, the number of internodes, distance between internodes and resistance to Fusarium wilt. The accession fiber quality was estimated by calculating stem slenderness, stem taperingness and ultimate fiber length. GWAS analysis was performed using the mixed linear model (MLM) with Principal component analysis (PCA) axes scores and Q matrix from analysis of population structure used as covariates for all phenotypic data. GWAS analysis identified a large number of genome intervals and potential gene candidates that may affect important agronomic traits.

Acknowledgments: This work is supported by the RSF grant 19-16-00030. 